\title{
An extreme ultraviolet wave associated with a failed eruption observed by the Solar Dynamics Observatory ${ }^{\star}$
}

\author{
R. Zheng, Y. Jiang, J. Yang, Y. Bi, J. Hong, B. Yang, and D. Yang \\ National Astronomical Observatories/Yunnan Astronomical Observatory, Chinese Academy of Sciences, Kunming 650011, PR China \\ e-mail: zhrsh@ynao.ac.cn
}

Received 20 October 2011 / Accepted 14 March 2012

\section{ABSTRACT}

\begin{abstract}
Aims. Taking advantage of the high temporal and spatial resolution of the Solar Dynamics Observatory (SDO) observations, we present an extreme ultraviolet (EUV) wave associated with a failed filament eruption that generated no coronal mass ejection (CME) on 2011 March 1. We aim at understanding the nature and origin of this EUV wave.

Methods. Combining the high-quality observations in the photosphere, the chromosphere, and the corona, we studied the characteristics of the wave and its relations to the associated eruption.

Results. The event occurred at an ephemeral region near a small active region. The continuous magnetic flux cancelation in the ephemeral region produced pre-eruption brightenings and two EUV jets, and excited the filament eruption, accompanying it with a microflare. After the eruption, the filament material appeared far from the eruption center, and the ambient loops seemed to be intact. It was evident that the filament eruption had failed and was not associated with a CME. The wave happened just after the north jet arrived, and apparently emanated ahead of the north jet, far from the eruption center. The wave propagated at nearly constant velocities in the range of $260-350 \mathrm{~km} \mathrm{~s}^{-1}$, with a slight negative acceleration in the last phase. Remarkably, the wave continued to propagate, and a loop in its passage was intact when wave and loop met.

Conclusions. Our analysis confirms that the EUV wave is a true wave, which we interpret as a fast-mode wave. In addition, the close temporal and spatial relationship between the wave and the jet provides evidence that the wave was likely triggered by the jet when the CME failed to happen.
\end{abstract}

Key words. Sun: activity - Sun: corona - Sun: filaments, prominences

\section{Introduction}

Extreme ultraviolet (EUV) waves were first observed with the Extreme ultraviolet Imaging Telescope (EIT; Delaboudinière et al. 1995) onboard the SOlar and Heliospheric Observatory (SOHO) spacecraft (e.g., Moses et al. 1997; Thompson et al. 1998), hence are originally referred to as "EIT waves". EUV waves generally appear as large-scale, diffuse, single-pulse coronal enhanced transients. They normally emanate from flaring and eruptive active regions and subsequently propagate over significant distances at speeds of several hundred $\mathrm{km} \mathrm{s}^{-1}$.

Hundreds of EUV waves were researched with various observations for more than a decade, but there are still some open questions. The controversy on the physical origin of EUV waves focuses on two possibilities: "flare-driven" and "coronal mass ejection (CME)-driven" (Biesecker et al. 2002; Cliver et al. 2005). Increasing evidence demonstrates that EUV waves are intimately associated with CMEs (or other types of mass motions) and not with flares (Biesecker et al. 2002; Cliver et al. 2005; Chen 2006). The generation of EUV waves by mass motions (most frequently by CMEs) is now widely accepted, and the controversy between the two generation mechanisms of EUV waves hardly exists anymore. Their nature is also under debate with competing models, such as coronal fast-mode magnetohydrodynamic (MHD) waves (e.g., Thompson et al. 1999; Wang 2000; Wu et al. 2001; Ofman \& Thompson 2002; Long et al. 2008; Gopalswamy et al. 2009; Veronig et al. 2010), slow-mode or

$\star$ Three movies are available in electronic form at http://www . aanda.org soliton-like waves (Wills-Davey et al. 2007; Wang et al. 2009), pseudo-waves related to a current shell or successive restructuring of magnetic field associated with the CME expansion (Delannée et al. 2008; Attrill et al. 2007; Chen et al. 2002), and hybrid models including both wave and pseudo-wave scenarios (Zhukov \& Auchère 2004; Cohen et al. 2009; Liu et al. 2010; Downs et al. 2011). For details of observations and models, we refer the reader to recent reviews (Wills-Davey \& Attrill 2009; Gallagher \& Long 2011; Warmuth 2010).

In this paper, with the high-cadence and sensitivity observations from the Atmospheric Imaging Assembly (AIA; Lemen et al. 2012) and the Helioseismic and Magnetic Imager (HMI) onboard the Solar Dynamics Observatory (SDO), the nature and origin of an EUV wave are studied in detail. We suggest that the wave is likely a fast-mode wave, which is potentially triggered by an EUV jet, not a CME or a flare.

\section{Observations and data analysis}

On 2011 March 1, an EUV wave emanated from an ephemeral region near a small active region (AR). It was associated with a failed filament eruption and EUV jets, and the eruption center is identified at the location of $x=52^{\prime \prime}, y=-172^{\prime \prime}$, measured from the solar disk center. We mainly used the observations from the SDO. The AIA has 10 EUV and UV wavelengths, covering a wide range of temperatures. The cadence is up to $12 \mathrm{~s}$, and the pixel resolution is 0 ". 6 . The HMI magnetograms are chosen to check the magnetic field configuration of the eruption 
region, with a cadence of $45 \mathrm{~s}$ and pixel resolution of 0 ". 6 . To check the filament morphology before its eruption, we used full-disk $\mathrm{H} \alpha$ filtergrams from the Global Oscillation Network Group (GONG) at the National Solar Observatory. In addition, the observations from the Extreme Ultraviolet Imager (EUVI; Howard et al. 2008) Ahead (-A) onboard the twin spacecraft Solar-TErrestrial RElations Observatory (STEREO; Kaiser et al. 2008) were used to study the evolution of the eruptions in the low corona. All images were individually rotated to a reference time (13:00:00 UT) to correct for solar rotation between images.

\section{Results}

\subsection{Failed filament eruption}

The left panels of Fig. 1 shows the $\mathrm{H} \alpha$ line center observations for the event before, during, and after the filament eruption. There were two clear filaments, marked as F1 and F2 in panel a. F1 was hook-like and about 30", the typical length of minifilament (Wang et al. 2000). At about 12:53 UT, there appeared brightenings around the crook of F1 (the black arrow in panel a), and F1 also became darker and thicker. Some minutes later, the filament began to erupt and resulted in the flaring patches on both sides of its southern segment (panel b). Meanwhile, F2 seemed to be unaffected (panel c), therefore it was weakly associated with the eruption. Remarkably, north of the eruption region, there appeared abundant cool material (arrows in panels $\mathrm{c}-\mathrm{d}$ ), staying there for about $20 \mathrm{~min}$ after the eruption. This indicated that the filament eruption was likely confined, and the mass was ejected to places far away, similar to the example in Liu et al. (2009). We also investigated the failed eruption in AIA $304 \AA$ images in the right panels. Before the eruption, only the southern segment of F1 was visible, and pre-eruption brightenings were clear around the crook of F1 (panel e). At about 02:56 UT, a jet emanated from the brightening site (panel f). Consistent with the situation in the $\mathrm{H} \alpha$ observations, there also appeared a dark patch north of the eruption region (the arrow in panel g), also likely an indicator of material falling off the eruptive filament. Of course, the explanation of falling material cannot eliminate other possibilities (e.g., new material cooling down from the corona or rising from the chromosphere). To track the evolution of the filament material in detail, a time-slice approach was employed. We chose a sector of 400" (S1 in panel e and $\mathrm{g}$ ), which is along the traveling direction of the jets from the eruption center (the plus at the south end of S1). Then we obtained slices along S1 from a series of AIA $304 \AA$ original images. Finally, we composed the slices over time to obtain a two-dimensional space-time plot, referred to as a time-slice image. In panel h, the dark patch was obvious (the left arrow), and the jets nearly stopped propagating at the edge of the dark patch. The falling material was clearly seen and lasted for about half an hour (right arrow).

In addition, we checked the failed eruption in 195 and $304 \AA$ images of EUVI-A in Fig. 2. The falling material of the filament eruption was very clear from the limb perspective, indicated by the arrows. The coronagraphs on the STEREO and SOHO did not detect any CME during the event, which further confirmed the failure of the filament eruption. Hence, it was credible that the dark patch in Fig. 1 represented the falling material from the failed filament eruption. The filament material was denser and cooler before the eruption. During the eruption, the material was ejected into a higher altitude, and became diffuse and heated. In the falling process, the material landed in a larger region.
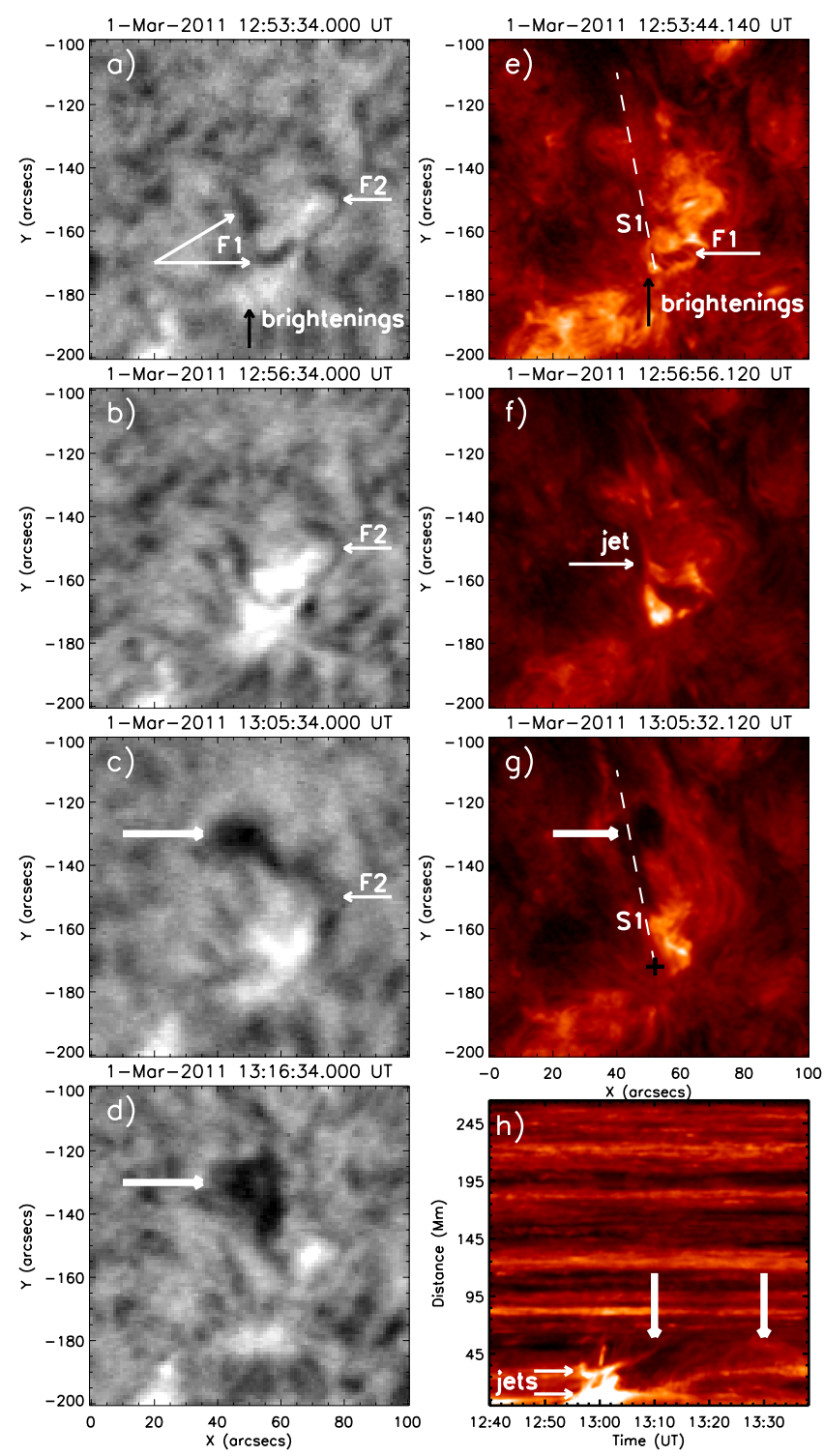

Fig. 1. Evolution of the filament before, during, and after the eruption in $\mathrm{H} \alpha$ filtergrams from the GONG/NSO (panels a)-d)) and in AIA 304 images (panels e)-g)). Panel h) is a time-slice image obtained along the sector S1, dashed lines in panels e) and g). F1 and F2 indicate two mini-filaments. The plus shows the eruption center. The thick arrows indicate the falling material of the erupting filament. The temporal evolution in the AIA $304 \AA$ as seen in the panels e)-g) is shown in the movie available in the online edition.

\subsection{Associated magnetic activities}

To fully understand the cause of the failed filament eruption, the associated magnetic activity is shown with HMI magnetograms in Fig. 3. The eruption region (the box in panel a) is located in the far west edge of a diffuse positive polarity, namely the leading sunspot of NOAA AR 11165. From about 13:00 UT on February 28, two bipoles successively emerged in about $20 \mathrm{~h}$ (panels $b-c)$. The positive-polarity leading spot (P1) of the west bipole was nearly fixed, and its negative-polarity following spot (N1) moved slowly southeastwards and became elongated. After the east bipole emerged, its positive-polarity leading spot (P2) moved westwards. As a result, N1 and P2 approached and collided with each other, hence the magnetic cancelation happened hours before the eruption onset and continued after 

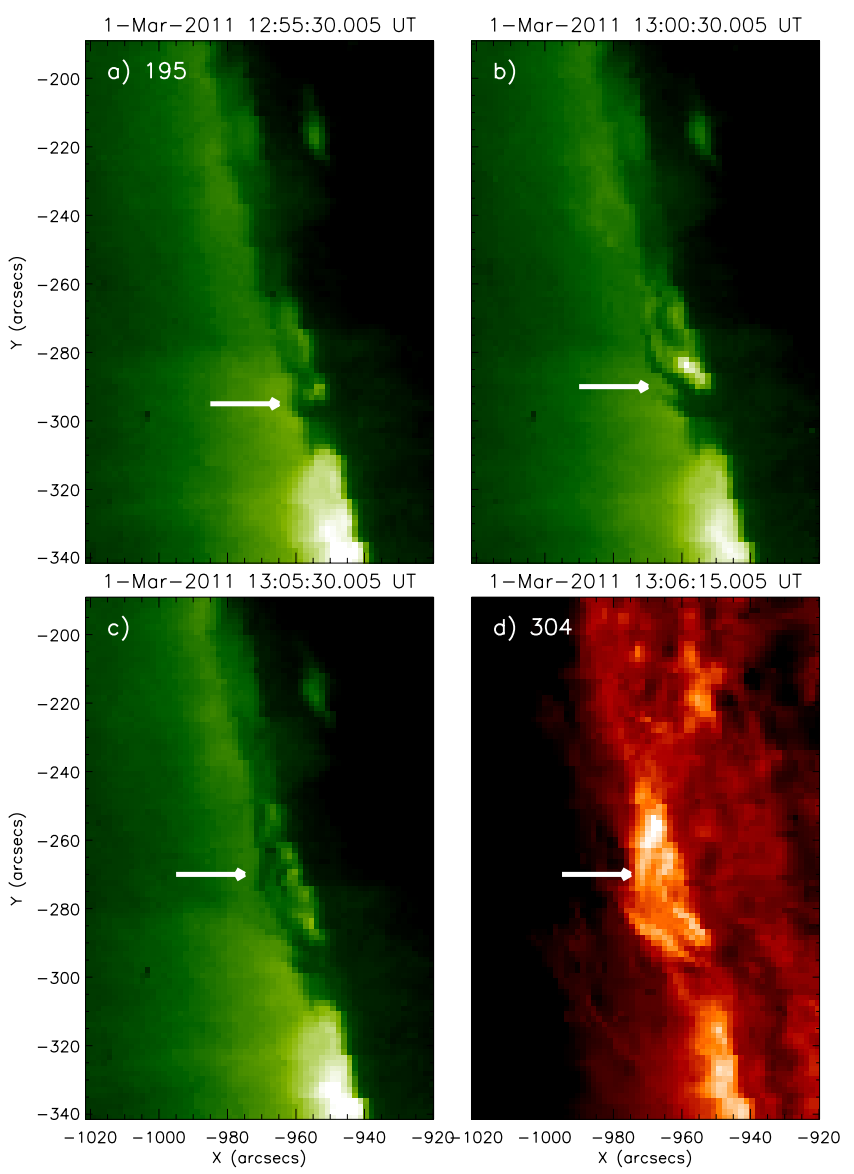

Fig. 2. Failed filament eruption shown in the original images in $195 \AA$ (panels a)-c)) and in $304 \AA$ (panel d)) of EUVI-A. The arrows indicate the movement of the filament.

the eruption. The eruption occurred at the region of the continuous magnetic cancelation between $\mathrm{N} 1$ and $\mathrm{P} 2$ (black boxes in panels $\mathrm{d}-\mathrm{g}$ ). In addition, there was a small magnetic cancelation region north of the eruption region (white boxes in panels $\mathrm{d}-\mathrm{g}$ ). In panel $\mathrm{h}$, the contour of $\mathrm{F} 1$ before the eruption is plotted over the magnetogram, in which the crook of the filament is located just at the cancelation site. Panel i plots the changes of the magnetic fluxes in the box region of panel $h$. There was a continuous flux cancelation from hours before the eruption to hours after the event end, the vertical line shows the eruption onset. In only $8 \mathrm{~h}$, the positive and negative fluxes both decreased by $7 \times 10^{19} \mathrm{Mx}$. It was suggested that the continuous magnetic cancelation could lead to a magnetic instability that triggered the filament eruption (e.g., van Ballegooijen \& Martens 1989; Amari et al. 2003).

\subsection{EUV jets and EUV wave}

The coronal response of the eruption is shown in the original images of AIA 171 and $193 \AA$ in Fig. 4. Before the eruption, the EUV filament was distinct (black arrows in panels $a-b$ ), and an S-shaped loops (L1) overlay its southern segment. There was a large-scale AR loop (L2) in the east close to the filament, and a straight loop (L3) in the north. Note that there were curved and sigmoid loops (L4 and L5) in the northwest. At about 12:54 UT, there appeared brightenings at the crook of the EUV filament channel (white arrows in panels a-b), cospatial with the $\mathrm{H} \alpha$ brightenings in Fig. 1, which implied the magnetic cancelation. About one minute later, an EUV jet (J1) emanated from the brightening site, and the filament began to erupt. It was interesting that there was another jet (J2) north of the eruption region (arrows in panels $\mathrm{c}-\mathrm{d}$ ), and the distance between the start points of the two jets was about $30 \mathrm{Mm}$. Namely, J2 just emanated from the north magnetic cancelation region indicated by white boxes in Fig. 3. Overlaid by the contours of the HMI magnetic field, it was clear that the jets both rooted in the magnetic cancelation region. It was probable that the magnetic cancelation produced the brightenings and EUV jets, as the precursor of the magnetic instability and runaway tether cutting, and eventually led to the filament eruption. As a result, the post-flare loops (PFLs) were formed above the filament channel (white arrows in panels e-f), consistent with the magnetic reconnection in the standard flare model (e.g., Kopp \& Pneuman 1976; Cargill \& Priest 1982). Particularly, obvious unwinding motion was observed during the eruption, in which the bright and dark loop structure intertwisted along the filament main axis (black arrows in panels e-f). It was consistent with the characteristic property of the helical kink instability of a twisted magnetic flux rope (e.g., Török \& Kliem 2003; Török et al. 2004; Kliem et al. 2004). After the eruption, L2, L4 and L5 were all still in their original places and seemed to be unaffected by the eruption, and only L3 was pushed aside by the eruption. The surviving loops could confirm that the filament eruption was confined by the large-scale overlying loops (i.e. L3), consistent with the remaining cool material in $\mathrm{H} \alpha$. In addition, the filament threads were still clearly seen above the eruption region about $20 \mathrm{~min}$ after the eruption (arrows in panels $\mathrm{g}-\mathrm{h}$ ), which provided another piece of evidence of the failed eruption.

Intriguingly, an EUV wave was formed during the eruption. The upper panels of Fig. 5 show the wave evolution in basedifference images of AIA $193 \AA$ A. J2 (the black arrow in panel b) reached a maximum length at about 13:00 UT. Ahead of J2, there appeared a faint bright patch as the wave front (the white arrow in panel b). As time went on, the front became brighter and propagated far away (white arrows in panels $\mathrm{c}-\mathrm{d}$ ). Note that a small sigmoid loop (L6) was right on the propagation passage of the wave and survived in the wave disturbance, which provided evidence that the wave was a true wave. In addition, the elongated coronal dimmings (black arrows in panels $\mathrm{c}-\mathrm{d}$ ) revealed that the failed eruption was confined by the loops, which were only pushed aside, not disrupted. To best display the EUV wave, we used the time-slice approach to analyze the kinematic evolution of the wave front in the bottom panels. Panel e shows the wave propagation along the traveling direction of the jets from the eruption center ( $\mathrm{S} 1$ in panel a, the same sector as that in Fig. 1). It was clear that the start point of $\mathrm{J} 2$ was about $30 \mathrm{Mm}$ removed from that of $\mathrm{J} 1$, consistent with the situation in the original images of Fig. 4. The following dimmings were likely due to the transverse displacement the loops pushed by the failed eruption. The speeds of $\mathrm{J} 1$ and $\mathrm{J} 2$ were $57 \pm 5$ and $151 \pm 9 \mathrm{~km} \mathrm{~s}^{-1}$, respectively. The wave front was weak and had an initial speed of $339 \pm 13 \mathrm{~km} \mathrm{~s}^{-1}$ and a final speed of $215 \pm 9 \mathrm{~km} \mathrm{~s}^{-1}$. The wave revealed an acceleration of $-156 \pm 23 \mathrm{~m} \mathrm{~s}^{-2}$ consistent with freely propagating fast-mode waves (Veronig et al. 2008, 2010; Long et al. 2008). The velocity, acceleration and associated errors of the wave front along $\mathrm{S} 1$ were derived by quadratic least-squares fits, assuming that the measurement uncertainty is 4 pixels $(\sim 1.74 \mathrm{Mm})$. The wave seemed to take off at about 13:00 UT, right after J2 arrived. Its start point seemed to be located at the brightenings (W) ahead of J2, about $80 \mathrm{Mm}$ far from the eruption center. Hence we identified $\mathrm{W}$ as the wave center, at about $x=25^{\prime \prime}, y=-70^{\prime \prime}$, and selected three sectors $(\mathrm{S} 2-\mathrm{S} 4$, dashed lines emanating from the wave center in 

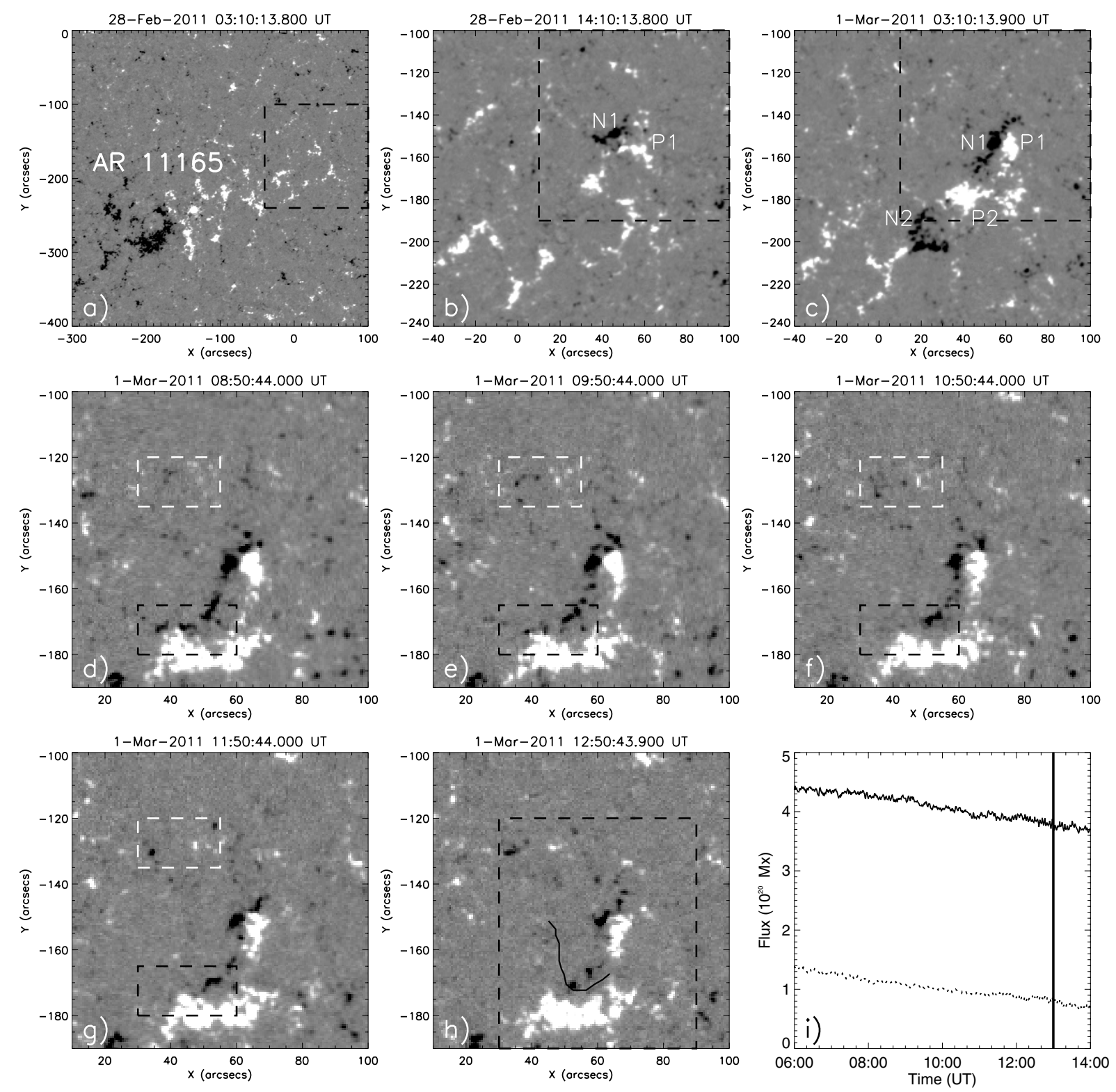

Fig. 3. Development of the emerging bipoles and magnetic cancelation in the eruption region by HMI magnetograms (panels a)-h)) and the magnetic flux evolution (panel i)) for the box region in panel $\mathbf{h}$ ), in which the contour of F1 is also superposed. The box in panel a) indicates the field of view (FOV) of panels $\mathbf{b})-\mathbf{c})$, in which the boxes show the FOV of panels $\mathbf{d}$ ) $-\mathbf{h}$ ). The boxes in panels d) $-\mathbf{g}$ ) indicate the cancelation region of the magnetic flux. The vertical line points out the eruption time.

panel b) to better show the wave propagation. The angles of $\mathrm{S} 2-\mathrm{S} 4$ are $25^{\circ}, 45^{\circ}$, and $55^{\circ}$, respectively, counted anticlockwise from the north. $\mathrm{S} 3$ was along the main direction of the wave propagation (namely, the north segment of dimmings), and S4 travel through the bright L6. The speeds of the wave in S2-S4 were about $296 \pm 10,348 \pm 19$, and $341 \pm 15 \mathrm{~km} \mathrm{~s}^{-1}$, respectively. The speeds and associated errors along S2-S4 were derived by linear fits, assuming the same measurement uncertainty as that for $\mathrm{S} 1$. The wave was followed by faint dimmings, which were most obvious in S3. In addition, the wave was associated with another distinct feature, stationary brightenings. It was interesting that the brightenings were far from the eruption center, not at the edge of the core dimmings like the situations in earlier works (Delannée et al. 2007). The lower brightenings at about $85 \mathrm{Mm}$ far from W along S4 were represented L6, which was bright before the wave arrival. Beyond the brightenings, the wave front still could be clearly distinguished, which was in favor of the fast-mode wave model.

\section{Discussions and conclusion}

Combining the observations from the SDO, the STEREO and the GONG/NSO, we presented an EUV wave associated with a failed filament eruption that generated no CME on 2011 March 1. Our main findings are as follows. (1) The continuous magnetic flux cancelation in an ephemeral region near a small AR produced pre-eruption brightenings and two EUV jets, as the indicator of the runaway tether cutting. (2) The magnetic cancelation led to a magnetic instability that triggered the filament eruption, accompanied by the microflare and PFLs. After the eruption, the filament material appeared at places far from the original sites; the filament threads were obvious in 

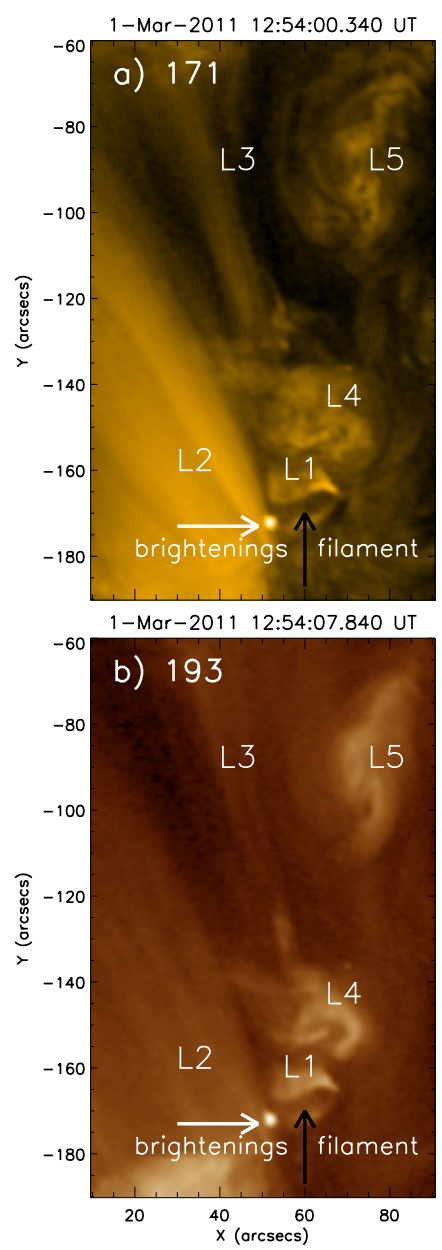
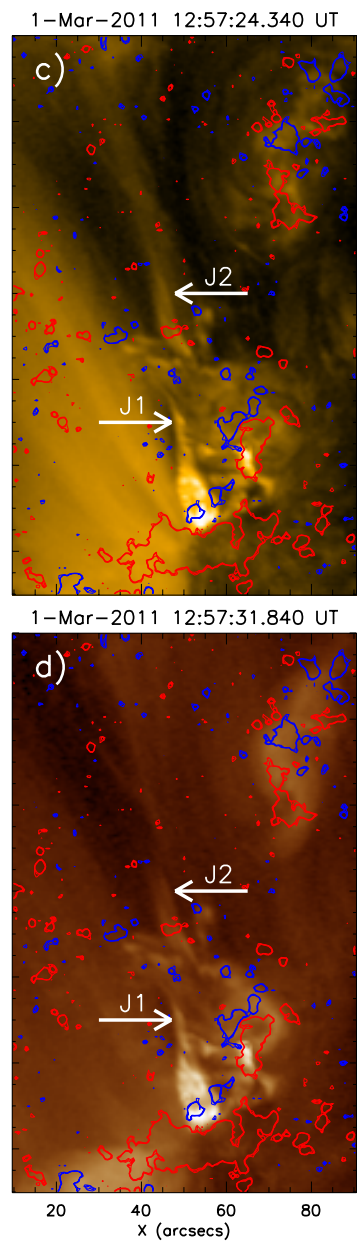
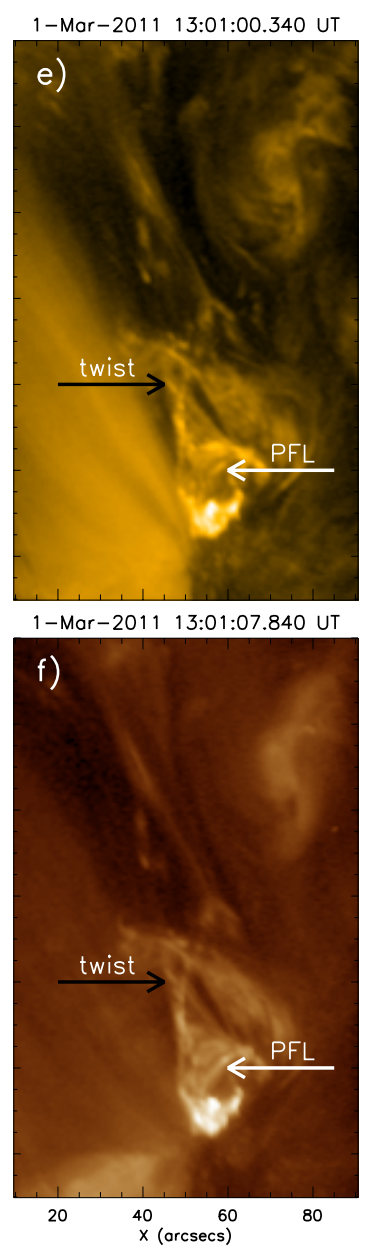
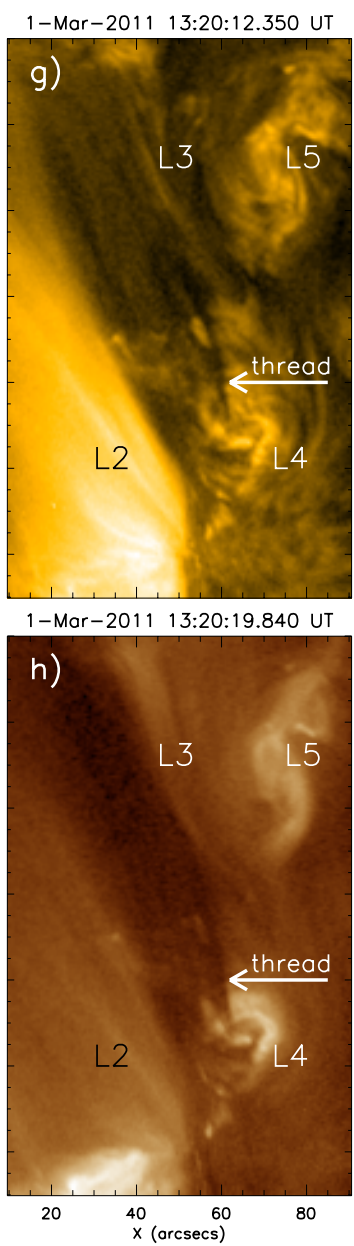

Fig. 4. Coronal structures associated with the eruption in the original images of AIA 171 (top panels) and $193 \AA$ (bottom panels). Contours of HMI longitudinal magnetic fields are also overlaid on the second column of images with positive fields in red and negative in blue. The levels are 20, 25, and 30 Gauss, respectively. L1-L5 denote the associated loops in the event. The temporal evolution in the AIA $171 \AA$ as seen in the top panels is shown in the movie available in the online edition.

EUV wavebands; the ambient loops also seemed to be intact. It was probable that the filament eruption was confined by the large-scale overlying magnetic filed, and the mass was drawn back down to the surface. (3) The wave happened just after the north jet arrived, and emanated ahead of the north jet, not from the eruption center. (4) The wave propagated at constant velocities in the range of $260-350 \mathrm{~km} \mathrm{~s}^{-1}$, with a slight negative acceleration in the last phase. Particularly, the wave continued to propagate, and the loop on its passage was intact when they encountered.

The wave speed is in the range of the average surfaceprojected expansion speeds for fast-mode waves (Wang 2000). The wave can hardly be explained in the slow-mode wave framework, in which the velocity cannot be higher than the coronal sound speed, that is about $160 \mathrm{~km} \mathrm{~s}^{-1}$ (Zhukov 2011). The negative acceleration character is consistent with the fast-mode waves, and the value is comparable to the range in previous works (Long et al. 2008, 2011; Veronig et al. 2008; Muhr et al. 2011). For the same event of 2007 May 19, Long et al. (2008) applied a numerical differencing technique to estimate an acceleration range of -413 to $816 \mathrm{~m} \mathrm{~s}^{-2}$; Veronig et al. (2008) derived an acceleration of $-160 \mathrm{~m} \mathrm{~s}^{-2}$ by fitting a quadratic model to distance-time measurements; Long et al. (2011) obtained an acceleration of $-256 \pm 134 \mathrm{~m} \mathrm{~s}^{-2}$ for the position of maximum intensity; Muhr et al. (2011) obtained an acceleration of $-85 \pm 60 \mathrm{~m} \mathrm{~s}^{-2}$. The kinematics of waves depends on the cadence of observations, and the method and chosen feature for the measurement (Long et al. 2008; Muhr et al. 2011). It was very likely that our result was derived by the time-slice approach with high temporal and spatial observations. In addition, the fact that L6 survived the wave passage might exclude the possibility that successive magnetic reconnections were driven by the flanks of CMEs (Attrill et al. 2007, 2009). On the other hand, the failure of the CME may exclude the field-line stretching model (Chen et al. 2002), in which the wave propagation needs the successive opening of magnetic field lines during the CME lift-off. Finally, the wave was associated with stationary brightenings, which are usually interpreted as an indication of non-wave nature (Delannée et al. 2007; Chen et al. 2005). The brightenings always form at the edge of core-coronal dimmings in the scenario, but those we studied were located far from the eruption center and appear to be instigated by the EUV wave. However, when an MHD wave crossed preexisting coronal structures (e.g. loops), it is capable of triggering a localized energy release that causes heating and a stationary emission enhancement (Wills-Davey \& Attrill 2009). To summarize, all results confirm that the EUV wave is a true wave, interpreted as a fast-mode wave.

Failed filament eruptions are particular events and have been observed in some cases (Moore et al. 2001; Ji et al. 2003; Liu et al. 2009; Shen et al. 2011). They generally are non-ejective and not associated with expelled CMEs. The filaments in the 

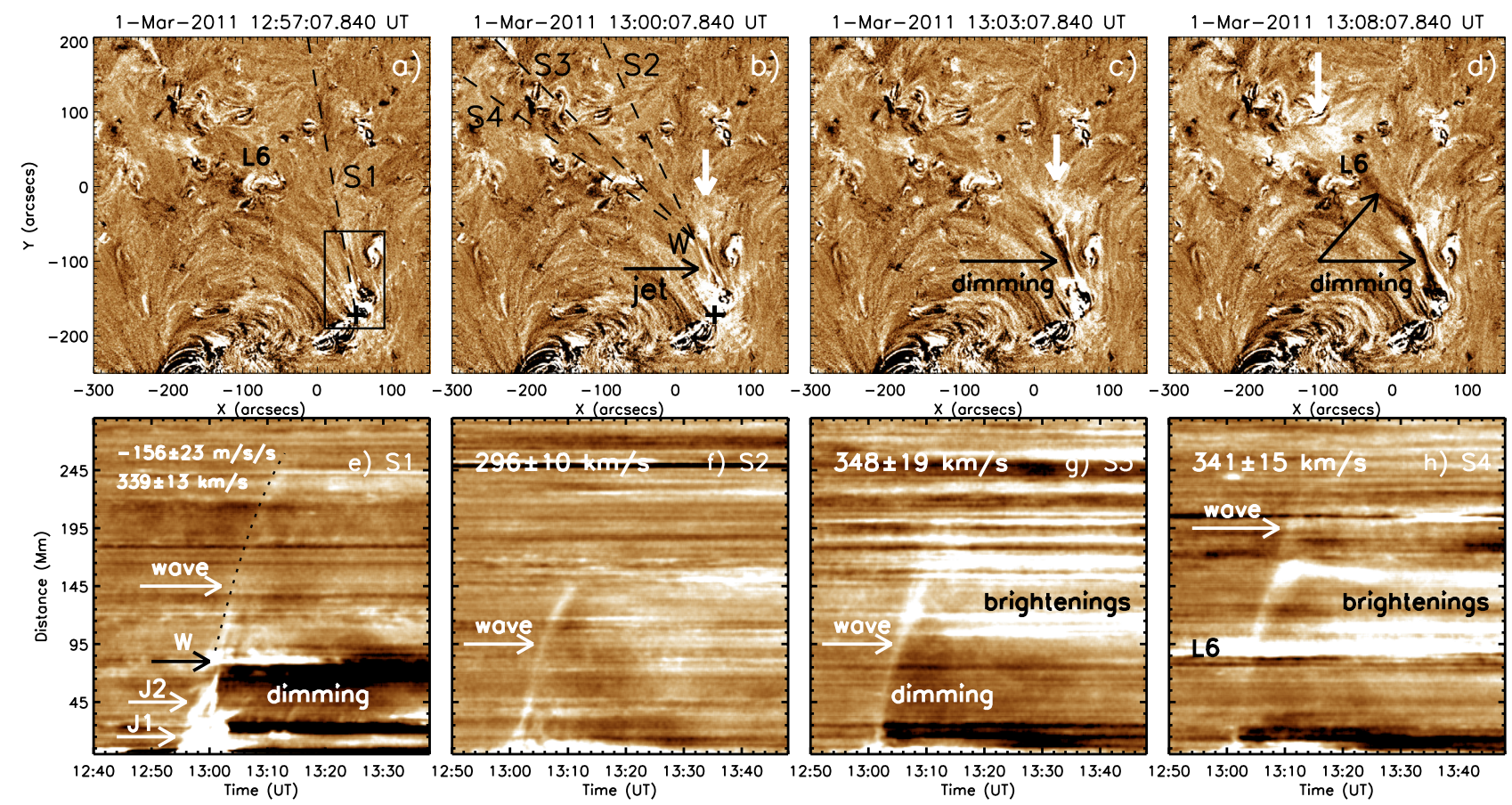

Fig. 5. Sequential base difference images in AIA $193 \AA$ (upper panels) displaying the evolution of the wave, and base difference time-slice images (bottom panels) along the sectors S1-S4 (dashed lines in panels a)-b)). S1 starts from the eruption center, indicated by the plus, and S2-S4 emanates from W. The box in panel a) shows the FOV of Fig. 4. Wave signatures are indicated by arrows, with the linear fitted velocities and quadratic fitted acceleration attached above. The temporal evolution in the AIA $193 \AA$ as seen in the upper panels is shown in the movie available in the online edition.

failed eruptions often display an initial acceleration, and the mass drains back down to the surface after it reaches a maximum height. Liu (2008) showed that the magnetic field's decrease with height is faster in a successful eruption than that in a failed eruption, and found that the field intensity at low altitude in a failed eruption was a few times stronger than that in a successful one. Liu et al. (2009) reported two failed filament eruptions in the asymmetric coronal fields, which could not only influence the trajectories of the ejecta, but also provided a relatively stronger confinement for flux rope eruptions. Similar to the event in Sterling et al. (2011), the filament eruption we studied was due to the gradual magnetic cancelation around it and confined by the large-scale overlying loops.

The physical origin of EUV waves is an active research topic. Biesecker et al. (2002) confirmed that EUV waves are strongly associated with CMEs and not with flares. Chen (2006) examined a set of strong flares (up to X-class X1.2) that probably produce strong pressure pulses, but did not have an associated CME. This author found that none of the flares in the sample were associated with EUV waves, and EUV waves and expanding dimmings appeared only when CMEs were present. Based on the catalog in Thompson \& Myers (2009), Cliver et al. (2005) concluded that a CME is the necessary condition for EUV wave creation. Attrill et al. (2009) found that a failed filament eruption produced neither the bright front of an EUV wave nor a CME, while a subsequent successful eruption from the same source region was associated with both; they reinforced the conclusion that a successful CME was necessary to generate a coronal wave. In our event, there was also no CME associated with the failed eruption, hence the wave cannot have been driven by a CME. On the other hand, the start point of the wave was far away from the microflare source. The different locations and weak intensity ruled out the flare as the wave driver. In addition, small-scale ejecta (e.g. spray surges, erupting plasmoids) could generate an initially driven shock, which travels freely after the ejection stops (Klein et al. 1999; Klassen et al. 2003). Naturally, owning to the close temporal and spatial relationship between the wave and $\mathbf{J} 2$, it was extremely probable that the wave was triggered by the jet, and continued to freely propagate after the jet stopped.

Furthermore, we stress that a CME and a failed eruption are very similar from a physical point of view. They both represent a mass motion that can produce a propagating fast magnetosonic wave, which may be observed as an EUV wave. But the failed filament eruption was not associated with any CME. There is a physical difference between mechanisms of wave generation by mass motions (related to CMEs or other eruptions) on the one hand, and by a thermal pressure pulse that may take place in flares on the other hand. But the difference between a wave initiated by a CME and a wave driven by another type of mass motion might be negligible from the physical point of view. Therefore, the jets may have produced the EUV wave in a way similar to the CME-driven mechanism, when the CME was absent (Vršnak \& Cliver 2008; Zhukov 2011).

However, the weak intensity of the EUV wave and associated eruption makes their nature uncertain and leaves other possibilities open. More observations of this type of the wave will be helpful to understand their nature and their relation with associated eruptions. The nature and origin of EUV waves remains unclear; more observations and theoretical work will be essential.

Acknowledgements. The authors thank the anonymous referee for constructive comments. The authors thank the SDO team, STEREO/EUVI team, and GONG/NSO consortium for providing the excellent data. This work is supported by the 973 Program (2011CB811403), and by the Natural Science Foundation of China under grants 10973038, 11173058, and 11103090 , by Shandong Province Natural Science Foundation ZR2011AQ009. 


\section{References}

Amari, T., Luciani, J. F., Aly, J. J., Mikic, Z., \& Linker, J. 2003, ApJ, 595, 1231 Attrill, G. D. R., Harra, L. K., van Driel-Gesztelyi, L., \& Démoulin, P. 2007, ApJ, 656, L101

Attrill, G. D. R., Engell, A., Wills-Davey, M. J., et al. 2009, ApJ, 704, 1296 Biesecker, D. A., Myers, D. C., Thompson, B. J., et al. 2002, ApJ, 569, 1009

Cargill, P. J., \& Priest, E. R. 1982, Sol. Phys., 76, 357

Chen, P. F. 2006, ApJ, 641, L153

Chen, P. F., Wu, S. T., Shibata, K., \& Fang, C. 2002, ApJ, 572, L99

Chen, P. F., Fang, C., \& Shibata, K. 2005, ApJ, 622, 1202

Cohen, O., Attrill, G. D. R., Manchester, W. B., IV, \& Wills-Davey, M. J. 2009, ApJ, 705, 587

Cliver, E. W., Laurenza, M., Storini, M., \& Thompson, B. J. 2005, ApJ, 631, 604 Delaboudinière, J.-P., Artzner, G. E., Brunaud, J., et al. 1995, Sol. Phys., 162, 291 Delannée, C., Hochedez, J. F., \& Aulanier, G. 2007, A\&A, 465, 203

Delannée, C., Török, T., Aulanier, G., \& Hochedez, J. F. 2008, Sol. Phys., 247, 123

Downs, C., Roussev, I. I., van der Holst, B., et al. 2011, ApJ, 728, 2

Gallagher, P. T., \& Long, D. M. 2011, Space Sci. Rev., 158, 365

Gopalswamy, N., Yashiro, S., Temmer, M., et al. 2009, ApJ, 691, L123

Howard, R. A., Moses, J. D., Vourlidas, A., et al. 2008, Space Sci. Rev., 136, 67 Ji, H., Wang, H., Schmahl, E. J., Moon, Y.-J., \& Jiang, Y. 2003, ApJ, 595, L135

Kaiser, M. L., Kucera, T. A., Davila, J. M., et al. 2008, Space Sci. Rev., 136, 5

Klassen, A., Pohjolainen, S., \& Klein, K.-L. 2003, Sol. Phys., 218, 197

Klein, K.-L., Khan, J. I., Vilmer, N., Delouis, J.-M., \& Aurass, H. 1999, A\&A, 346, L53

Kliem, B., Titov, V. S., \& Török, T. 2004, A\&A, 413, L23

Kopp, R. A., \& Pneuman, G. W. 1976, Sol. Phys., 50, 85

Lemen, J. R., Title, A. M., Akin, D. J., et al. 2012, Sol. Phys., 275, 17

Liu, Y. 2008, ApJ, 679, L151

Liu, Y., Su, J., Xu, Z., et al. 2009, ApJ, 696, L70

Liu, W., Nitta, N. V., Schrijver, C. J., Title, A. M., \& Tarbell, T. D. 2010, ApJ, 723, L53
Long, D. M., Gallagher, P. T., McAteer, R. T. J., \& Bloomfield, D. S. 2008, ApJ, 680, L81

Long, D. M., Gallagher, P. T., McAteer, R. T. J., \& Bloomfield, D. S. 2011, A\&A, 531, A42

Moore, R. L., Sterling, A. C., Hudson, H. S., \& Lemen, J. R. 2001, ApJ, 552, 833

Moses, D., Clette, F., Delaboudinière, J.-P., et al. 1997, Sol. Phys., 175, 571

Muhr, N., Veronig, A. M., Kienreich, I. W., Temmer, M., \& Vršnak, B. 2011, ApJ, 739, 89

Ofman, L., \& Thompson, B. J. 2002, ApJ, 574, 440

Shen, Y. D., Liu, Y., \& Liu, R. 2011, Res. Astron. Astrophys., 11, 594

Sterling, A. C., Moore, R. L., \& Freeland, S. L. 2011, ApJ, 731, L3

van Ballegooijen, A. A., \& Martens, P. C. H. 1989, ApJ, 343, 971

Veronig, A. M., Temmer, M., \& Vršnak, B. 2008, ApJ, 681, L113

Veronig, A. M., Muhr, N., Kienreich, I. W., Temmer, M., \& Vršnak, B. 2010, ApJ, 716, L157

Vršnak, B., \& Cliver, E. W. 2008, Sol. Phys., 253, 215

Thompson, B. J., \& Myers, D. C. 2009, ApJS, 183, 225

Thompson, B. J., Plunkett, S. P., Gurman, J. B., et al. 1998, Geophys. Res. Lett., 25,2465

Thompson, B. J., Gurman, J. B., Neupert, W. M., et al. 1999, ApJ, 517, L151

Török, T., \& Kliem, B. 2003, A\&A, 406, 1043

Török, T., Kliem, B., \& Titov, V. S. 2004, A\&A, 413, L27

Wang, Y. M. 2000, ApJ, 543, L89

Wang, J., Li, W., Denker, C., et al. 2000, ApJ, 530, 1071

Wang, H., Shen, C., \& Lin, J. 2009, ApJ, 700, 1716

Warmuth, A. 2010, Adv. Space Res., 45, 527

Wills-Davey, M. J., \& Attrill, G. D. R. 2009, Space Sci. Rev., 149, 325

Wills-Davey, M. J., DeForest, C. E., \& Stenflo, J. O. 2007, ApJ, 664, 556

Wu, S. T., Zheng, H. N., Wang, S., et al. 2001, J. Geophys. Res., 106, 25089

Zhukov, A. N. 2011, JASTP, 73, 1096

Zhukov, A. N., \& Auchère, F. 2004, A\&A, 427, 705 